\title{
The error analysis of Crank-Nicolson-type difference scheme for fractional subdiffusion equation with spatially variable coefficient
}

Pu Zhang ${ }^{1,2}$ and Hai Pu ${ }^{1,3^{*}}$

"Correspondence:
puhaicumt@163.com
'State Key Laboratory for
Geomechanics and Deep
Underground Engineering, China
University of Mining and
Technology, Xuzhou, Jiangsu
221116, P.R. China
${ }^{3}$ School of Mechanics and Civil
Engineering, China University of
Mining and Technology, Xuzhou,
Jiangsu 221116, P.R. China
Full list of author information is
available at the end of the article

${ }^{\text {"Correspondence: }}$

puhaicumt@163.com

${ }^{1}$ State Key Laboratory for

Geomechanics and Deep

Underground Engineering, China

University of Mining and

221116, P.R. China

${ }^{3}$ School of Mechanics and Civil Engineering, China University of Jiangsu 221116, P.R. China

available at the end of the article

\begin{abstract}
A Crank-Nicolson-type difference scheme is presented for the spatial variable coefficient subdiffusion equation with Riemann-Liouville fractional derivative. The truncation errors in temporal and spatial directions are analyzed rigorously. At each time level, it results in a linear system in which the coefficient matrix is tridiagonal and strictly diagonally dominant, so it can be solved by the Thomas algorithm. The unconditional stability and convergence of the scheme are proved in the discrete $L_{2}$ norm by the energy method. The convergence order is $\min \left\{2-\frac{\alpha}{2}, 1+\alpha\right\}$ in the temporal direction and two in the spatial one. Finally, numerical examples are presented to verify the efficiency of our method.
\end{abstract}

MSC: 65M06;65M12;65M15

Keywords: fractional subdiffusion equation; variable coefficient; finite difference; stability; convergence

\section{Introduction}

In recent years, fractional differential equations have captured great attention of research in different domains. This facts reflect the ability of fractional calculation to describe many phenomena in different disciplines such as semiconductors, mechanics, signal processing, porous media, anomalous diffusion, and so on [1-6]. Employing fractional derivatives to describe the procedure of anomalous diffusion, we get the time fractional subdiffusion equation $[2,7,8]$ :

$$
\frac{\partial u(x, t)}{\partial t}={ }_{0} \mathcal{D}_{t}^{1-\alpha}\left[K_{r} \frac{\partial^{2} u(x, t)}{\partial x^{2}}\right]+f(x, t)
$$

where ${ }_{0} \mathcal{D}_{t}^{1-\alpha}(0<\alpha<1)$ denotes the Riemann-Liouville fractional derivative operator defined as

$$
{ }_{0} \mathcal{D}_{t}^{1-\alpha} u(t)=\frac{1}{\Gamma(\alpha)} \frac{d}{d t} \int_{0}^{t} \frac{u(s)}{(t-s)^{1-\alpha}} d s .
$$

Some researchers considered the similar form with Caputo derivative:

$$
{ }_{0}^{C} \mathcal{D}_{t}^{\alpha} u(x, t)=\frac{\partial^{2} u(x, t)}{\partial x^{2}}+g(x, t)
$$

(c) The Author(s) 2017. This article is distributed under the terms of the Creative Commons Attribution 4.0 International License (http://creativecommons.org/licenses/by/4.0/), which permits unrestricted use, distribution, and reproduction in any medium, provided you give appropriate credit to the original author(s) and the source, provide a link to the Creative Commons license, and indicate if changes were made. 
where ${ }_{0}^{C} \mathcal{D}_{t}^{\alpha}$ denotes Caputo's derivative operator defined by

$$
{ }_{0}^{C} \mathcal{D}_{t}^{\alpha} u(t)=\frac{1}{\Gamma(1-\alpha)} \int_{0}^{t} \frac{d u(s)}{d s}(t-s)^{-\alpha} d s, \quad \alpha \in(0,1) .
$$

We have the following relation between the Caputo and Riemann-Liouville fractional derivatives.

Let $y(t)$ be an $(m-1)$ times continuously differentiable function in the interval $[0, T]$ with $y^{(m)}(t)$ integrable in $[0, T]$. For every $p$, if $0 \leq m-1 \leq p \leq m$, then the Riemann-Liouville fractional derivative ${ }_{0} \mathcal{D}_{t}^{p} y(t)$ exists, and the following equality holds [1]:

$$
\begin{aligned}
{ }_{0} \mathcal{D}_{t}^{p} y(t) & =\sum_{j=0}^{m-1} \frac{y^{(j)}(0) t^{j-p}}{\Gamma(1+j-p)}+{ }_{0}^{C} \mathcal{D}_{t}^{p} y(t) \\
& =\sum_{j=0}^{m-1} \frac{y^{(j)}(0) t^{j-p}}{\Gamma(1+j-p)}+\frac{1}{\Gamma(m-p)} \int_{0}^{t} \frac{y^{(m)}(s)}{(t-s)^{p-m+1}} d s .
\end{aligned}
$$

Much remarkable work has been done theoretically for diffusion and fractional problems [9-11]. Marin and Marinescu [10] studied the asymptotic partition of total energy for the solutions of the mixed initial boundary value problem within the context of the thermoelasticity of initially stressed bodies, and Hameed et al. [11] derived and analyzed a mathematical model subject to low Reynolds number and long wavelength approximations in order to study the peristaltic motion of fractional second-grade fluid in a vertical tube. In the regard of numerical work for time-fractional diffusion equations, Langlands and Henry [12] obtained an implicit numerical method for the homogeneous problem and discussed the accuracy and stability of their scheme. Zhuang et al. [13] integrated the linear and nonlinear subdiffusion equations about the time variable $t$ and then approximated the obtained equivalent equations numerically with the idea of numerical integrals. Yuste and Acedo [14] developed an explicit scheme and gave a strict proof of the stability of the explicit scheme, and then Yuste [15] analyzed the weighted average finite difference scheme by the von Neumann method.

One main approximation approach to a discrete analog of the time-fractional derivative is an $L_{1}$ formula. Sun and Wu [16] first derived a fully discrete difference scheme employing the $L_{1}$ approximation, where the truncation error was proved to be of order $2-\alpha$ in temporal accuracy. Lin and $\mathrm{Xu}$ [17] constructed an effective numerical method by employing the finite difference scheme in time and using the Legendre spectral methods in space. Chen et al. [18] gave an implicit numerical scheme for the problem and proved the unconditional stability and $L_{2}$-norm convergence. Gao and Sun [19] applied the $L_{1}$ formula to approximate the Caputo time-fractional derivative and developed a compact finite difference scheme to promote the spatial accuracy for the fractional subdiffusion equation. They obtained the fourth-order convergence rate in spatial direction. Another main way to a discrete analog of the fractional derivative is the shifted Grünwald-Letnikov formula. Very recently, Deng's group $[20,21]$ has presented a high-order discrete analog of the space-fractional derivative by assembling the shifted GL operator with different weights.

The Crank-Nicolson difference scheme is a classical method for difference approximation. The works employing the $\mathrm{CN}$ method for fractional problems constantly emerge. Zhang et al. [22] presented a Crank-Nicolson-type difference scheme for a subdiffusion 
equation with Riemann-Liouville fractional derivative, where the discrete $H_{1}$ norm convergence was proved rigorously, and the maximum norm error estimate was given. Based on a Crank-Nicolson-type discretization, Wang and Vong [23] proposed a second-order accuracy formula to approximate the time-fractional derivative and established a compact finite difference scheme for solving the modified anomalous fractional subdiffusion equation. For more applications of the Crank-Nicolson scheme, we refer the reader to [24-26].

The works we listed are mostly focused on the subdiffusion equation with constant coefficient. However, many practical applications involved variable diffusion coefficients [2729]. For example, the flow of heat in a rod is constituted by composite heat-conducting materials, which means that the diffusion coefficient may vary with space variable. In view of some external heat source, Zhao [30] considered the Caputo-fractional subdiffusion equation with spatially variable coefficient:

$$
{ }_{0}^{C} \mathcal{D}_{t}^{\alpha} u(x, t)=\frac{\partial}{\partial x}\left(\varphi(x) \frac{\partial u}{\partial x}\right)+g(x, t) .
$$

Employing an $L_{1}$ formula, she obtained the convergence of order $2-\alpha$ in the temporal direction and fourth-order approximation order in space. Vong et al. [31] considered the same equation under Neumann boundary conditions and obtained the global convergence of order $O\left(\tau^{2-\alpha}+h^{4}\right)$. Metzler et al. [32] suggested the following fractional model equation for anomalous diffusion:

$$
{ }_{0} \mathcal{D}_{t}^{\frac{2}{d_{w}}} P(r, t)=\frac{1}{r^{d_{s}-1}} \frac{\partial}{\partial r}\left(r^{d_{s}-1} \frac{\partial P(r, t)}{\partial r}\right), \quad r>0, t>0,
$$

where $P(r, t)$ is the probability density of random walks on fractals, $d_{w}>2$ is the anomalous diffusion exponent, $d_{s}$ is just the spectral dimension of the fractal, and $d_{s}=\frac{2 d_{f}}{d_{w}}$ with $d_{f}$ denoting fractal dimension of the underlying object. Employing (5) and neglecting the coefficient $\frac{1}{r^{d s}-1}$ (which has no impact on difference approximation), (7) can be transformed into equation (6).

If $u(x, t)$ is suitably smooth in time, then we have the following relationship [1,33]:

$$
{ }_{0} \mathcal{D}_{t}^{1-\alpha}\left[{ }_{0}^{C} \mathcal{D}_{t}^{\alpha} u(x, t)\right]=\frac{\partial u(x, t)}{\partial t} .
$$

Therefore, implementing the operator ${ }_{0} \mathcal{D}_{t}^{1-\alpha}$ on both sides of (6), we derive the following Riemann-Liouville fractional subdiffusion equation with spatially variable coefficient:

$$
\frac{\partial u(x, t)}{\partial t}={ }_{0} \mathcal{D}_{t}^{1-\alpha}\left[\frac{\partial}{\partial x}\left(\varphi(x) \frac{\partial u}{\partial x}\right)\right]+f(x, t)
$$

where $f(x, t)={ }_{0} \mathcal{D}_{t}^{1-\alpha} g(x, t)$.

From the preceding discussion we see that equation (9) is a more general form. In this paper, we consider the difference scheme of (9). As far as we know, the difference scheme for this equation has not been proposed by now. In the present work, we establish a CrankNicolson-type difference scheme for the spatial variable coefficient problem (9) by the discrete Riemann-Liouville fractional derivative with $L_{1}$ formula. The CN-type scheme results in a linear system in which the coefficient matrix is tridiagonal and strictly diagonally dominant, so the Thomas algorithm suits. 
The content of this paper is organized as follows. In Section 2, we introduce essential notation and some preliminary lemmas and then construct the Crank-Nicolson-type finite difference scheme. The unique solvability, unconditional stability, and the $L_{2}$-norm convergence are proved in Section 3 by the energy method. Some examples are listed in Section 4 to verify our theoretical analysis and testify the validation of our difference scheme. Finally, a brief conclusion ends this work.

\section{Derivation of a $\mathrm{CN}$-type difference scheme}

Consider the following subdiffusion equation with spatially variable coefficient combined with initial boundary value conditions:

$$
\begin{aligned}
& \frac{\partial u(x, t)}{\partial t}={ }_{0} \mathcal{D}_{t}^{1-\alpha}\left[\frac{\partial}{\partial x}\left(\varphi(x) \frac{\partial u}{\partial x}\right)\right]+f(x, t), \quad 0<x<L, 0<t \leq T, \\
& u(0, t)=\Phi_{1}(t), \quad u(L, t)=\Phi_{2}(t), \quad 0<t \leq T, \\
& u(x, 0)=\Psi(x), \quad 0 \leq x \leq L,
\end{aligned}
$$

where $0<\alpha<1$, and we suppose that $c_{1} \leq \varphi(x) \leq c_{2}$ and $\varphi(x), f(x, t), \Phi_{1}(t), \Phi_{2}(t), \Psi(x)$ are sufficiently smooth functions.

For a finite difference approximation, we suppose that $M$ and $N$ are two positive integers and let $h=\frac{L}{M}$ and $\tau=\frac{T}{N}$ be space and temporal step lengths, respectively. Define $x_{i}=$ $i h, 0 \leq i \leq M, t_{n}=n \tau, 0 \leq n \leq N, \Omega_{h}=\left\{x_{i} \mid 0 \leq i \leq M\right\}, \Omega_{\tau}=\left\{t_{n} \mid 0 \leq n \leq N\right\}$, and, in addition, $t_{k-\frac{1}{2}}=\left(k-\frac{1}{2}\right) \tau, x_{i-\frac{1}{2}}=\left(i-\frac{1}{2}\right) h$.

For any grid function $u=\left\{u_{i}^{n} \mid 0 \leq i \leq M, 0 \leq n \leq N\right\}$, we denote

$$
\begin{array}{ll}
\delta_{x} u_{i-\frac{1}{2}}^{n}=\frac{1}{h}\left(u_{i}^{n}-u_{i-1}^{n}\right), & \delta_{x}^{2} u_{i}^{n}=\frac{1}{h}\left(\delta_{x} u_{i+\frac{1}{2}}^{n}-\delta_{x} u_{i-\frac{1}{2}}^{n}\right), \\
u_{i}^{n-\frac{1}{2}}=\frac{1}{2}\left(u_{i}^{n}+u_{i}^{n-1}\right), \quad \delta_{t} u_{i}^{n-\frac{1}{2}}=\frac{1}{\tau}\left(u_{i}^{n}-u_{i}^{n-1}\right) .
\end{array}
$$

The following lemmas are needed for our error analysis.

Lemma 1 ([16]) For $0<\alpha<1$ and $y \in C^{2}\left[0, t_{n}\right]$, we have:

$$
\begin{aligned}
& \frac{1}{\Gamma(\alpha)} \int_{0}^{t} \frac{y^{\prime}(s) d s}{\left(t_{n}-s\right)^{\alpha}}-\frac{\tau^{\alpha-1}}{\Gamma(1+\alpha)}\left[y\left(t_{n}\right)-\sum_{k=1}^{n-1}\left(a_{n-k-1}-a_{n-k}\right) y\left(t_{k}\right)-a_{n-1} y(0)\right] \\
& \quad=\sum_{k=1}^{n} \int_{t_{k-1}}^{t_{k}} L_{\tau, \alpha, n}(s) y^{\prime \prime}(s) d s=O\left(\tau^{1+\alpha}\right),
\end{aligned}
$$

where $a_{k}=(k+1)^{\alpha}-k^{\alpha}$, and for $s \in\left(t_{k-1}, t_{k}\right)$,

$$
L_{\tau, \alpha, n}(s)=\frac{1}{\Gamma(1+\alpha)}\left\{\left(t_{n}-s\right)^{\alpha}-\left[\frac{s-t_{k-1}}{\tau}\left(t_{n}-t_{k}\right)^{\alpha}+\frac{t_{k}-s}{\tau}\left(t_{n}-t_{k-1}\right)^{\alpha}\right]\right\} .
$$

Furthermore,

$$
\sum_{k=1}^{n} \int_{t_{k-1}}^{t_{k}} L_{\tau, \alpha, n}(s) d s \leq \frac{1}{\Gamma(1+\alpha)}\left[\frac{\alpha}{12}+\frac{2^{1+\alpha}}{1+\alpha}-\left(1+2^{\alpha-1}\right)\right] \tau^{1+\alpha} .
$$


Lemma 2 ([34]) Let $y \in C^{3}\left[t_{k-1}, t_{k}\right]$. Then

$$
\begin{aligned}
\frac{1}{2}\left[y^{\prime}\left(t_{k}\right)+y^{\prime}\left(t_{k-1}\right)\right]= & \frac{1}{\tau}\left[y\left(t_{k}\right)-y\left(t_{k-1}\right)\right] \\
& +\frac{\tau^{2}}{16} \int_{0}^{1}\left[y^{(3)}\left(t_{k-\frac{1}{2}}+\frac{s \tau}{2}\right)+y^{(3)}\left(t_{k-\frac{1}{2}}-\frac{s \tau}{2}\right)\right]\left(1-s^{2}\right) d s .
\end{aligned}
$$

Now we define the grid function $U_{i}^{n}=u\left(x_{i}, t_{n}\right), 0 \leq i \leq M, 0 \leq n \leq N$, and, in addition, denote $\varphi\left(x_{i+\frac{1}{2}}\right)$ by $\varphi_{i+\frac{1}{2}}$.

Lemma 3 Suppose $u \in C^{4}\left[x_{i}, x_{i+1}\right]$. Then

$$
\begin{aligned}
\delta_{x} U_{i+\frac{1}{2}}= & u^{\prime}\left(x_{i+\frac{1}{2}}\right)+\frac{h}{4} \int_{0}^{1}\left[u^{\prime \prime}\left(x_{i+\frac{1}{2}}+\frac{h}{2} t\right)-u^{\prime \prime}\left(x_{i+\frac{1}{2}}-\frac{h}{2} t\right)\right](1-t) d t, \\
\delta_{x} U_{i+\frac{1}{2}}= & u^{\prime}\left(x_{i+\frac{1}{2}}\right)+\frac{h^{2}}{16} \int_{0}^{1}\left[u^{(3)}\left(x_{i+\frac{1}{2}}+\frac{h}{2} t\right)+u^{(3)}\left(x_{i+\frac{1}{2}}-\frac{h}{2} t\right)\right](1-t)^{2} d t, \\
\delta_{x} U_{i+\frac{1}{2}}= & u^{\prime}\left(x_{i+\frac{1}{2}}\right)+\frac{h^{3}}{96} \int_{0}^{1}\left[u^{(4)}\left(x_{i+\frac{1}{2}}+\frac{h}{2} t\right)-u^{(4)}\left(x_{i+\frac{1}{2}}-\frac{h}{2} t\right)\right](1-t)^{3} d t \\
& +\frac{h^{2}}{24} u^{(3)}\left(x_{i+\frac{1}{2}}\right) .
\end{aligned}
$$

Proof Employing the Taylor expansion with integral remainder, we have:

$$
\begin{aligned}
& U_{i+1}=u\left(x_{i+\frac{1}{2}}\right)+\frac{h}{2} u^{\prime}\left(x_{i+\frac{1}{2}}\right)+\frac{h^{2}}{2} \int_{0}^{1} u^{\prime \prime}\left(x_{i+\frac{1}{2}}+\frac{t h}{2}\right)(1-t) d t \\
& U_{i}=u\left(x_{i+\frac{1}{2}}\right)-\frac{h}{2} u^{\prime}\left(x_{i+\frac{1}{2}}\right)+\frac{h^{2}}{2} \int_{0}^{1} u^{\prime \prime}\left(x_{i+\frac{1}{2}}-\frac{t h}{2}\right)(1-t) d t .
\end{aligned}
$$

Then subtracting these two equalities, we get the first statement. The proofs of the other two are similar by using an expansion of higher order.

Now we analyze the truncation error of the $L_{1}$ analog for the Riemann-Liouville fractional derivative.

Lemma 4 Let $u(x, t) \in C^{4,2}([0, L] \times[0, T]), \varphi(x) \in C^{1}[0, L]$. Then for the truncation error, we have:

$$
\begin{aligned}
{ }_{0} \mathcal{D}_{t}^{1-\alpha} & {\left[\frac{\partial}{\partial x}\left(\varphi(x) \frac{\partial u}{\partial x}\right)\right]\left(x_{i}, t_{n}\right) } \\
= & \frac{\tau^{\alpha-1}}{\Gamma(1+\alpha)}\left[\delta_{x}\left(\varphi \delta_{x} U\right)_{i}^{n}-\sum_{k=1}^{n-1}\left(a_{n-k-1}-a_{n-k}\right) \delta_{x}\left(\varphi \delta_{x} U\right)_{i}^{k}-a_{n-1} \delta_{x}\left(\varphi \delta_{x} U\right)_{i}^{0}\right] \\
& +\frac{t_{n}^{\alpha-1}}{\Gamma(\alpha)} \delta_{x}\left(\varphi \delta_{x} U\right)_{i}^{0}+\left(R_{1}\right)_{i}^{n}, \quad 1 \leq i \leq M-1,1 \leq n \leq N
\end{aligned}
$$


where $\left(R_{1}\right)_{i}^{n}=\left(R_{11}\right)_{i}^{n}+\left(R_{12}\right)_{i}^{n}$, and

$$
\begin{aligned}
\left|\left(R_{11}\right)_{i}^{n}\right| \leq & \frac{1}{24} \max _{\substack{0 \leq x \leq L \\
0 \leq t \leq T}}\left[\left|{ }_{0} \mathcal{D}_{t}^{1-\alpha} \frac{\partial^{3}}{\partial x^{3}}\left(\varphi \frac{\partial u}{\partial x}\right)\right|+\left|{ }_{0} \mathcal{D}_{t}^{1-\alpha} \frac{\partial}{\partial x}\left(\varphi \frac{\partial^{3} u}{\partial x^{3}}\right)\right|\right. \\
& \left.+\frac{1}{4}\left|\varphi(x)_{0} \mathcal{D}_{t}^{1-\alpha} \frac{\partial^{4} u}{\partial x^{4}}\right|\right] h^{2}, \\
\left|\left(R_{12}\right)_{i}^{n}\right| \leq & \frac{1}{\Gamma(1+\alpha)}\left[\frac{\alpha}{12}+\frac{2^{1+\alpha}}{1+\alpha}-\left(1+2^{\alpha-1}\right)\right] \\
& \max _{\substack{0 \leq x \leq L \\
0 \leq t \leq T}}\left[\left|\frac{\partial^{3}}{\partial x \partial t^{2}}\left(\varphi(x) \frac{\partial u}{\partial x}\right)\right|+\frac{1}{2}\left|\varphi(x) \frac{\partial^{4} u}{\partial x^{2} \partial t^{2}}\right|\right] \tau^{1+\alpha} .
\end{aligned}
$$

Proof Let ${ }_{0} \mathcal{D}_{t}^{1-\alpha} u=w$ and $W_{i}^{n}=w\left(x_{i}, t_{n}\right)$. Then

$$
{ }_{0} \mathcal{D}_{t}^{1-\alpha}\left[\frac{\partial}{\partial x}\left(\varphi(x) \frac{\partial u}{\partial x}\right)\right]=\frac{\partial}{\partial x}\left(\varphi(x) \frac{\partial w}{\partial x}\right) .
$$

It follows from Lemma 3 that

$$
\begin{aligned}
\delta_{x}\left(\varphi \delta_{x} W\right)_{i}^{n}= & \frac{1}{h}\left(\varphi_{i+\frac{1}{2}} \delta_{x} W_{i+\frac{1}{2}}^{n}-\varphi_{i-\frac{1}{2}} \delta_{x} W_{i-\frac{1}{2}}^{n}\right) \\
= & \frac{1}{h}\left(\varphi_{i+\frac{1}{2}} w_{x}^{\prime}\left(x_{i+\frac{1}{2}}, t_{n}\right)-\varphi_{i-\frac{1}{2}} w_{x}^{\prime}\left(x_{i-\frac{1}{2}}, t_{n}\right)\right) \\
& +\frac{h^{2}}{24} \frac{1}{h}\left[\varphi_{i+\frac{1}{2}} w_{x}^{(3)}\left(x_{i+\frac{1}{2}}, t_{n}\right)-\varphi_{i-\frac{1}{2}} w_{x}^{(3)}\left(x_{i-\frac{1}{2}}, t_{n}\right)\right] \\
& +\varphi_{i+\frac{1}{2}} \frac{h^{2}}{96} \int_{0}^{1}\left[w_{x}^{(4)}\left(x_{i+\frac{1}{2}}+\frac{t}{2} h\right)-w_{x}^{(4)}\left(x_{i+\frac{1}{2}}-\frac{t}{2} h\right)\right](1-t)^{3} d t \\
& -\varphi_{i-\frac{1}{2}} \frac{h^{2}}{96} \int_{0}^{1}\left[w_{x}^{(4)}\left(x_{i-\frac{1}{2}}+\frac{t}{2} h\right)-w_{x}^{(4)}\left(x_{i-\frac{1}{2}}-\frac{t}{2} h\right)\right](1-t)^{3} d t \\
= & \frac{\partial}{\partial x}\left(\varphi(x) \frac{\partial w}{\partial x}\right)\left(x_{i}, t_{n}\right)+\frac{h^{2}}{24}\left(\varphi w_{x}^{(3)}\right)_{x}^{\prime}\left(\xi, t_{n}\right) \\
& +\frac{h^{2}}{16} \int_{0}^{1}\left[\left(\varphi w_{x}^{\prime}\right)_{x}^{(3)}\left(x_{i}+\frac{t}{2} h, t_{n}\right)+\left(\varphi w_{x}^{\prime}\right)_{x}^{(3)}\left(x_{i}-\frac{t}{2} h, t_{n}\right)\right](1-t)^{2} d t \\
& +\varphi_{i+\frac{1}{2}} \frac{h^{2}}{96} \int_{0}^{1}\left[w_{x}^{(4)}\left(x_{i+\frac{1}{2}}+\frac{t}{2} h, t_{n}\right)-w_{x}^{(4)}\left(x_{i+\frac{1}{2}}-\frac{t}{2} h, t_{n}\right)\right](1-t)^{3} d t \\
& -\varphi_{i-\frac{1}{2}} \frac{h^{2}}{96} \int_{0}^{1}\left[w_{x}^{(4)}\left(x_{i-\frac{1}{2}}+\frac{t}{2} h, t_{n}\right)-w_{x}^{(4)}\left(x_{i-\frac{1}{2}}-\frac{t}{2} h, t_{n}\right)\right] \\
& \cdot(1-t)^{3} d t,
\end{aligned}
$$

where $\xi \in\left[x_{i-1}, x_{i+1}\right]$. So we have

$$
\frac{\partial}{\partial x}\left(\varphi(x) \frac{\partial w}{\partial x}\right)\left(x_{i}, t_{n}\right)=\delta_{x}\left(\varphi \delta_{x} W\right)_{i}^{n}+\left(R_{11}\right)_{i}^{n}
$$


where

$$
\begin{aligned}
\left|\left(R_{11}\right)_{i}^{n}\right| \leq & \frac{1}{24} \max _{\substack{0 \leq x \leq L \\
0 \leq t \leq T}}\left[\left|{ }_{0} \mathcal{D}_{t}^{1-\alpha} \frac{\partial^{3}}{\partial x^{3}}\left(\varphi \frac{\partial u}{\partial x}\right)\right|+\left|{ }_{0} \mathcal{D}_{t}^{1-\alpha} \frac{\partial}{\partial x}\left(\varphi \frac{\partial^{3} u}{\partial x^{3}}\right)\right|\right. \\
& \left.+\frac{1}{4}\left|\varphi(x)_{0} \mathcal{D}_{t}^{1-\alpha} \frac{\partial^{4} u}{\partial x^{4}}\right|\right] h^{2} .
\end{aligned}
$$

It follows from (15) and (5) that

$$
\begin{aligned}
\delta_{x}\left(\varphi \delta_{x} W\right)_{i}^{n}= & {\left[{ }_{0} \mathcal{D}_{t}^{1-\alpha} \delta_{x}\left(\varphi \delta_{x} U\right)\right]\left(x_{i}, t_{n}\right) } \\
= & \frac{\tau^{\alpha-1}}{\Gamma(1+\alpha)}\left[\delta_{x}\left(\varphi \delta_{x} U\right)_{i}^{n}-\sum_{k=1}^{n-1}\left(a_{n-k-1}-a_{n-k}\right) \delta_{x}\left(\varphi \delta_{x} U\right)_{i}^{k}-a_{n-1} \delta_{x}\left(\varphi \delta_{x} U\right)_{i}^{0}\right] \\
& +\frac{t_{n}^{\alpha-1}}{\Gamma(\alpha)} \delta_{x}\left(\varphi \delta_{x} U\right)_{i}^{0}+\sum_{k=1}^{n} \int_{t_{k-1}}^{t_{k}} L_{\tau, \alpha, n}(s) \frac{\partial^{2}}{\partial t^{2}} \delta_{x}\left(\varphi \delta_{x} u\right)\left(x_{i}, s\right) d s .
\end{aligned}
$$

Using similar analysis and applying Lemma 3 again, we get

$$
\begin{aligned}
\delta_{x}\left(\varphi \delta_{x} U\right)_{i}^{n}= & \frac{1}{2} \int_{0}^{1}\left[\frac{\partial}{\partial x}\left(\varphi(x) \frac{\partial u}{\partial x}\right)\left(x_{i}+\frac{t}{2} h, t_{n}\right)+\frac{\partial}{\partial x}\left(\varphi(x) \frac{\partial u}{\partial x}\right)\left(x_{i}-\frac{t}{2} h, t_{n}\right)\right] d t \\
& +\frac{1}{4} \varphi_{i+\frac{1}{2}} \int_{0}^{1}\left[\frac{\partial^{2} u}{\partial x^{2}}\left(x_{i+\frac{1}{2}}+\frac{t}{2} h, t_{n}\right)-\frac{\partial^{2} u}{\partial x^{2}}\left(x_{i+\frac{1}{2}}-\frac{t}{2} h, t_{n}\right)\right](1-t) d t \\
& -\frac{1}{4} \varphi_{i-\frac{1}{2}} \int_{0}^{1}\left[\frac{\partial^{2} u}{\partial x^{2}}\left(x_{i-\frac{1}{2}}+\frac{t}{2} h, t_{n}\right)-\frac{\partial^{2} u}{\partial x^{2}}\left(x_{i-\frac{1}{2}}-\frac{t}{2} h, t_{n}\right)\right](1-t) d t .
\end{aligned}
$$

Applying (17) and the last equality, it is not hard to get

$$
\begin{aligned}
\left|\left(R_{12}\right)_{i}^{n}\right| \leq & \frac{1}{\Gamma(1+\alpha)}\left[\frac{\alpha}{12}+\frac{2^{1+\alpha}}{1+\alpha}-\left(1+2^{\alpha-1}\right)\right] \\
& \max _{\substack{0 \leq x \leq L \\
0 \leq t \leq T}}\left[\left|\frac{\partial^{3}}{\partial x \partial t^{2}}\left(\varphi(x) \frac{\partial u}{\partial x}\right)\right|+\frac{1}{2}\left|\varphi(x) \frac{\partial^{4} u}{\partial x^{2} \partial t^{2}}\right|\right] \tau^{1+\alpha} .
\end{aligned}
$$

The proof is completed.

We now construct a Crank-Nicolson-type scheme for problem (10)-(12). Considering equality (11) at the point $\left(x_{i}, t_{n}\right)$, we have

$$
\frac{\partial u\left(x_{i}, t_{n}\right)}{\partial t}={ }_{0} \mathcal{D}_{t}^{1-\alpha}\left[\frac{\partial}{\partial x}\left(\varphi(x) \frac{\partial u}{\partial x}\right)\right]\left(x_{i}, t_{n}\right)+f\left(x_{i}, t_{n}\right), \quad 1<n<N, 1<i<M-1 .
$$

Then

$$
\begin{aligned}
\frac{1}{2}[ & \left.\frac{\partial u\left(x_{i}, t_{n}\right)}{\partial t}+\frac{\partial u\left(x_{i}, t_{n-1}\right)}{\partial t}\right] \\
= & \frac{1}{2}\left\{{ }_{0} \mathcal{D}_{t}^{1-\alpha}\left[\frac{\partial}{\partial x}\left(\varphi(x) \frac{\partial u}{\partial x}\right)\right]\left(x_{i}, t_{n}\right)+{ }_{0} \mathcal{D}_{t}^{1-\alpha}\left[\frac{\partial}{\partial x}\left(\varphi(x) \frac{\partial u}{\partial x}\right)\right]\left(x_{i}, t_{n-1}\right)\right\} \\
& +\frac{1}{2}\left[f\left(x_{i}, t_{n}\right)+f\left(x_{i}, t_{n-1}\right)\right] .
\end{aligned}
$$


From Lemma 2 we obtain

$$
\frac{1}{2}\left[\frac{\partial u\left(x_{i}, t_{n}\right)}{\partial t}+\frac{\partial u\left(x_{i}, t_{n-1}\right)}{\partial t}\right]=\delta_{t} U_{i}^{n-\frac{1}{2}}+\left(R_{2}\right)_{i}^{n}
$$

where

$$
\left(R_{2}\right)_{i}^{n}=\frac{\tau^{2}}{16} \int_{0}^{1}\left[\frac{\partial^{3} u}{\partial t^{3}}\left(x_{i}, t_{n-\frac{1}{2}}+\frac{s \tau}{2}\right)+\frac{\partial^{3} u}{\partial t^{3}}\left(x_{i}, t_{n-\frac{1}{2}}-\frac{s \tau}{2}\right)\right]\left(1-s^{2}\right) d s
$$

It follows from Lemma 4 that

$$
\begin{aligned}
& \frac{1}{2}\left\{{ }_{0} \mathcal{D}_{t}^{1-\alpha}\left[\frac{\partial}{\partial x}\left(\varphi(x) \frac{\partial u}{\partial x}\right)\right]\left(x_{i}, t_{n}\right)+{ }_{0} \mathcal{D}_{t}^{1-\alpha}\left[\frac{\partial}{\partial x}\left(\varphi(x) \frac{\partial u}{\partial x}\right)\right]\left(x_{i}, t_{n-1}\right)\right\} \\
& =\frac{t_{n}^{\alpha-1}+t_{n-1}^{\alpha-1} \delta_{x}\left(\varphi \delta_{x} U\right)_{i}^{0}}{2 \Gamma(\alpha)} \\
& \quad+\frac{\tau^{\alpha-1}}{2 \Gamma(1+\alpha)}\left[\delta_{x}\left(\varphi \delta_{x} U\right)_{i}^{n}-\sum_{k=1}^{n-1}\left(a_{n-k-1}-a_{n-k}\right) \delta_{x}\left(\varphi \delta_{x} U\right)_{i}^{k}-a_{n-1} \delta_{x}\left(\varphi \delta_{x} U\right)_{i}^{0}\right] \\
& +\frac{\tau^{\alpha-1}}{2 \Gamma(1+\alpha)}\left[\delta_{x}\left(\varphi \delta_{x} U\right)_{i}^{n-1}-\sum_{k=1}^{n-2}\left(a_{n-k-2}-a_{n-k-1}\right) \delta_{x}\left(\varphi \delta_{x} U\right)_{i}^{k}-a_{n-2} \delta_{x}\left(\varphi \delta_{x} U\right)_{i}^{0}\right] \\
& +\frac{1}{2}\left(R_{1}\right)_{i}^{n}+\frac{1}{2}\left(R_{1}\right)_{i}^{n-1} .
\end{aligned}
$$

Denoting $U_{i}^{n-\frac{1}{2}}=\frac{1}{2}\left(U_{i}^{n}+U_{i}^{n-1}\right)$ and $\delta_{x}\left(\varphi \delta_{x} U\right)_{i}^{n-\frac{1}{2}}=\frac{1}{2}\left[\delta_{x}\left(\varphi \delta_{x} U\right)_{i}^{n}+\delta_{x}\left(\varphi \delta_{x} U\right)_{i}^{n-1}\right]$ and noticing that

$$
\begin{aligned}
& -\sum_{k=1}^{n-2}\left(a_{n-k-2}-a_{n-k-1}\right) \delta_{x}\left(\varphi \delta_{x} U\right)_{i}^{k}-a_{n-2} \delta_{x}\left(\varphi \delta_{x} U\right)_{i}^{0} \\
& =-\sum_{l=2}^{n-1}\left(a_{n-l-1}-a_{n-l}\right) \delta_{x}\left(\varphi \delta_{x} U\right)_{i}^{l-1}-a_{n-2} \delta_{x}\left(\varphi \delta_{x} U\right)_{i}^{0} \\
& =-\sum_{l=1}^{n-1}\left(a_{n-l-1}-a_{n-l}\right) \delta_{x}\left(\varphi \delta_{x} U\right)_{i}^{l-1}+\left(a_{n-2}-a_{n-1}\right) \delta_{x}\left(\varphi \delta_{x} U\right)_{i}^{0}-a_{n-2} \delta_{x}\left(\varphi \delta_{x} U\right)_{i}^{0} \\
& =-\sum_{k=1}^{n-1}\left(a_{n-k-1}-a_{n-k}\right) \delta_{x}\left(\varphi \delta_{x} U\right)_{i}^{k-1}-a_{n-1} \delta_{x}\left(\varphi \delta_{x} U\right)_{i}^{0}
\end{aligned}
$$

we have

$$
\begin{aligned}
\frac{1}{2}\left\{{ }_{0} \mathcal{D}_{t}^{1-\alpha}\left\{\frac{\partial}{\partial x}\left[\varphi(x) \frac{\partial u}{\partial x}\right]\right\}\left(x_{i}, t_{n}\right)+{ }_{0} \mathcal{D}_{t}^{1-\alpha}\left\{\frac{\partial}{\partial x}\left[\varphi(x) \frac{\partial u}{\partial x}\right]\right\}\left(x_{i}, t_{n-1}\right)\right\} \\
=\frac{t_{n}^{\alpha-1}+t_{n-1}^{\alpha-1} \delta_{x}\left(\varphi \delta_{x} U\right)_{i}^{0}+\frac{1}{2}\left(R_{1}\right)_{i}^{n}+\frac{1}{2}\left(R_{1}\right)_{i}^{n-1}+\frac{\tau^{\alpha-1}}{2 \Gamma(1+\alpha)}}{2 \Gamma(\alpha)} \\
\cdot\left[\delta_{x}\left(\varphi \delta_{x} U\right)_{i}^{n}-\sum_{k=1}^{n-1}\left(a_{n-k-1}-a_{n-k}\right) \delta_{x}\left(\varphi \delta_{x} U\right)_{i}^{k}-a_{n-1} \delta_{x}\left(\varphi \delta_{x} U\right)_{i}^{0}\right]+\frac{\tau^{\alpha-1}}{2 \Gamma(1+\alpha)}
\end{aligned}
$$




$$
\begin{aligned}
& \cdot\left[\delta_{x}\left(\varphi \delta_{x} U\right)_{i}^{n-1}-\sum_{k=1}^{n-1}\left(a_{n-k-1}-a_{n-k}\right) \delta_{x}\left(\varphi \delta_{x} U\right)_{i}^{k-1}-a_{n-1} \delta_{x}\left(\varphi \delta_{x} U\right)_{i}^{0}\right] \\
= & \frac{t_{n}^{\alpha-1}+t_{n-1}^{\alpha-1}}{2 \Gamma(\alpha)} \delta_{x}\left(\varphi \delta_{x} U\right)_{i}^{0}+\left(R_{1}\right)_{i}^{n-\frac{1}{2}}+\frac{\tau^{\alpha-1}}{\Gamma(1+\alpha)} \\
& \cdot\left[\delta_{x}\left(\varphi \delta_{x} U\right)_{i}^{n-\frac{1}{2}}-\sum_{k=1}^{n-1}\left(a_{n-k-1}-a_{n-k}\right) \delta_{x}\left(\varphi \delta_{x} U\right)_{i}^{k-\frac{1}{2}}-a_{n-1} \delta_{x}\left(\varphi \delta_{x} U\right)_{i}^{0}\right] .
\end{aligned}
$$

Substituting (40) and (36) into (35), we have

$$
\begin{aligned}
\delta_{t} U_{i}^{n-\frac{1}{2}}= & \frac{\tau^{\alpha-1}}{\Gamma(1+\alpha)}\left[\delta_{x}\left(\varphi \delta_{x} U\right)_{i}^{n-\frac{1}{2}}-\sum_{k=1}^{n-1}\left(a_{n-k-1}-a_{n-k}\right) \delta_{x}\left(\varphi \delta_{x} U\right)_{i}^{k-\frac{1}{2}}\right. \\
& \left.-a_{n-1} \delta_{x}\left(\varphi \delta_{x} U\right)_{i}^{0}\right]+\frac{t_{n}^{\alpha-1}+t_{n-1}^{\alpha-1}}{2 \Gamma(\alpha)} \delta_{x}\left(\varphi \delta_{x} U\right)_{i}^{0}+f_{i}^{n-\frac{1}{2}}+(R)_{i}^{n}
\end{aligned}
$$

where $(R)_{i}^{n}=\left(R_{1}\right)_{i}^{n-\frac{1}{2}}-\left(R_{2}\right)_{i}^{n}, 1 \leq i \leq M-1,2 \leq n \leq N$.

When $n=1$, using equality (34) at the point $\left(x_{i}, t_{1}\right)$ and employing the Taylor expansion, we have

$$
\begin{aligned}
\delta_{t} U_{i}^{\frac{1}{2}} & =\frac{\partial u}{\partial t}\left(x_{i}, t_{1}\right)-\left(R_{3}\right)_{i} \\
& ={ }_{0} \mathcal{D}_{t}^{1-\alpha} \frac{\partial}{\partial x}\left[\varphi(x) \frac{\partial u}{\partial x}\right]\left(x_{i}, t_{1}\right)+f\left(x_{i}, t_{1}\right)-\left(R_{3}\right)_{i},
\end{aligned}
$$

where

$$
\left(R_{3}\right)_{i}=\tau \int_{0}^{1} \frac{\partial^{2} u}{\partial t^{2}}\left(x_{i}, s \tau\right) s d s
$$

Using Lemma 4, we arrive at

$$
\begin{aligned}
\delta_{t} U_{i}^{\frac{1}{2}}= & \frac{\tau^{\alpha-1}}{\Gamma(1+\alpha)}\left[\delta_{x}\left(\varphi \delta_{x} U\right)_{i}^{1}-a_{0} \delta_{x}\left(\varphi \delta_{x} U\right)_{i}^{0}\right]+\frac{\tau^{\alpha-1}}{\Gamma(\alpha)} \delta_{x}\left(\varphi \delta_{x} U\right)_{i}^{0} \\
& +f\left(x_{i}, t_{1}\right)+\left(R_{1}\right)_{i}^{1}-\left(R_{3}\right)_{i} \\
= & \frac{\tau^{\alpha-1}}{\Gamma(1+\alpha)}\left[\delta_{x}\left(\varphi \delta_{x} U\right)_{i}^{1}+(\alpha-1) \delta_{x}\left(\varphi \delta_{x} U\right)_{i}^{0}\right]+f_{i}^{1}+R_{i},
\end{aligned}
$$

where

$$
R_{i}=\left(R_{1}\right)_{i}^{1}-\left(R_{3}\right)_{i}, \quad 1 \leq i \leq M-1 .
$$

By the previous analysis there exists a constant $C$, independent of $h$ and $\tau$, satisfying

$$
\begin{aligned}
& \left|R_{i}^{n}\right| \leq C\left(\tau^{1+\alpha}+h^{2}\right), \quad 1 \leq i \leq M-1,2 \leq n \leq N, \\
& \left|R_{i}\right| \leq C\left(\tau+h^{2}\right), \quad 1 \leq i \leq M-1 .
\end{aligned}
$$


The initial and boundary conditions can be written as

$$
\begin{aligned}
& U_{0}^{n}=\Phi_{1}\left(t_{n}\right), \quad U_{M}^{n}=\Phi_{2}\left(t_{n}\right), \quad 1 \leq n \leq N \\
& U_{i}^{0}=\Psi\left(x_{i}\right), \quad 0 \leq i \leq M
\end{aligned}
$$

Ignoring the truncation errors $R_{i}^{n}$ in (41) and $R_{i}$ in (44) and replacing the grid function $U_{i}^{n}$ with its numerical analog $u_{i}^{n}$, we arrive at the following difference scheme:

$$
\begin{aligned}
& \delta_{t} u_{i}^{n-\frac{1}{2}}= \frac{\tau^{\alpha-1}}{\Gamma(1+\alpha)}\left[\delta_{x}\left(\varphi \delta_{x} u\right)_{i}^{n-\frac{1}{2}}-\sum_{k=1}^{n-1}\left(a_{n-k-1}-a_{n-k}\right) \delta_{x}\left(\varphi \delta_{x} u\right)_{i}^{k-\frac{1}{2}}-a_{n-1} \delta_{x}\left(\varphi \delta_{x} u\right)_{i}^{0}\right] \\
& \quad+\frac{t_{n}^{\alpha-1}+t_{n-1}^{\alpha-1}}{2 \Gamma(\alpha)} \delta_{x}\left(\varphi \delta_{x} u\right)_{i}^{0}+f_{i}^{n-\frac{1}{2}}, \quad 1 \leq i \leq M-1,2 \leq n \leq N, \\
& \delta_{t} u_{i}^{\frac{1}{2}}= \frac{\tau^{\alpha-1}}{\Gamma(1+\alpha)}\left[\delta_{x}\left(\varphi \delta_{x} u\right)_{i}^{1}+(\alpha-1) \delta_{x}\left(\varphi \delta_{x} u\right)_{i}^{0}\right]+f_{i}^{1}, \quad 1 \leq i \leq M-1, \\
& u_{0}^{n}=\Phi_{1}\left(t_{n}\right), \quad u_{M}^{n}=\Phi_{2}\left(t_{n}\right), \quad 1 \leq n \leq N, \\
& u_{i}^{0}=\Psi\left(x_{i}\right), \quad 0 \leq j \leq M .
\end{aligned}
$$

It is easy to see that, at each time level, the difference scheme (49)-(52) is a tridiagonal system with strictly diagonal dominant coefficient matrix, and thus the difference scheme has a unique solution, and the Thomas algorithm suits.

\section{Analysis of stability and convergence of the $\mathrm{CN}$-type difference scheme}

\subsection{Stability}

Now we introduce necessary notation and lemmas, which will be used in the analysis of stability and convergence.

Define the grid function space $\mathcal{S}_{h}=\left\{u \mid u=\left(u_{0}, u_{1}, \ldots, u_{M}\right), u_{0}=u_{M}=0\right\}$ on $\Omega_{h}$. For any $v, w \in \mathcal{S}_{h}$, we define the discrete inner products and corresponding norms as follows:

$$
\begin{aligned}
& (v, w)_{h}=h \sum_{i=1}^{M-1} v_{i} w_{i}, \quad\|v\|=\sqrt{(v, v)_{h}}, \\
& \langle v, w\rangle=h \sum_{i=0}^{M-1}\left(\delta_{x} v_{i+\frac{1}{2}}\right)\left(\delta_{x} w_{i+\frac{1}{2}}\right), \quad\left\|\delta_{x} v\right\|=\sqrt{\langle v, v\rangle}, \\
& \left(\delta_{x} u, \delta_{x} v\right)_{\varphi}=h \sum_{i=0}^{M-1} \varphi\left(x_{i+\frac{1}{2}}\right)\left(\delta_{x} u_{i+\frac{1}{2}}\right)\left(\delta_{x} v_{i+\frac{1}{2}}\right), \quad\left\|\delta_{x} u\right\|_{\varphi}=\sqrt{\left(\delta_{x} u, \delta_{x} u\right)_{\varphi}}, \\
& \|v\|_{\infty}=\max _{0 \leq i \leq M}\left|v_{i}\right| .
\end{aligned}
$$

Considering the smoothness of $\varphi(x)$, it is not hard to get

$$
\sqrt{c_{1}}\left\|\delta_{x} u\right\| \leq\left\|\delta_{x} u\right\|_{\varphi} \leq \sqrt{c}_{2}\left\|\delta_{x} u\right\|
$$

Lemma 5 ([35]) For any grid function $u \in \mathcal{S}_{h}$,

$$
\|u\| \leq \frac{L}{\sqrt{6}}\left\|\delta_{x} u\right\| .
$$


We have the following properties of $a_{n}$.

\section{Lemma 6}

$$
\begin{aligned}
& 1=a_{0}>a_{1}>a_{2} \cdots>a_{n} \rightarrow 0, \\
& \alpha(n+1)^{\alpha-1}<a_{n}<\alpha n^{\alpha-1}, \\
& a_{n-1}<\frac{1}{2}\left[\alpha n^{\alpha-1}+\alpha(n-1)^{\alpha-1}\right], \quad n \geq 2, \\
& \hat{a}_{n-1}<a_{n-1}, \quad \hat{a}_{n-1}<a_{n-2}-a_{n-2}, \quad n \geq 2,
\end{aligned}
$$

where $\hat{a}_{n-1}=\frac{1}{2}\left[\alpha n^{\alpha-1}+\alpha(n-1)^{\alpha-1}\right]-a_{n-1}$.

Proof Noticing that $a_{n}=\alpha \int_{n}^{n+1} x^{\alpha-1} d x$ and $x^{\alpha-1}$ is a strictly convex and decreasing function, the first three relations hold.

From inequalities (54)-(56) we have

$$
\begin{aligned}
4 a_{n-1}-\left[\alpha n^{\alpha-1}+\alpha(n-1)^{\alpha-1}\right] & >3 \alpha n^{\alpha-1}-\alpha(n-1)^{\alpha-1} \\
& =\alpha n^{\alpha-1}\left[3-\left(\frac{n}{n-1}\right)^{1-\alpha}\right]>0
\end{aligned}
$$

and

$$
a_{n-2}>\alpha(n-1)^{\alpha-1}>\frac{1}{2}\left[\alpha n^{\alpha-1}+\alpha(n-1)^{\alpha-1}\right]
$$

so that (57) holds.

We now give the proof of the stability of the difference scheme (49)-(52) with respect to the initial value $u_{i}^{0}$ and the inhomogeneous term $f$. We denote

$$
f^{1}=\left(0, f_{1}^{1}, f_{2}^{1}, \ldots, f_{M-1}^{1}, 0\right)
$$

and

$$
f^{n-\frac{1}{2}}=\left(0, f_{1}^{n-\frac{1}{2}}, f_{2}^{n-\frac{1}{2}}, \ldots, f_{M-1}^{n-\frac{1}{2}}, 0\right), \quad 2 \leq n \leq N .
$$

Theorem 1 Let $u_{i}^{m}, 0 \leq i \leq M, 1 \leq m \leq N$, be a solution of the finite difference scheme (49)-(52) with $\Psi_{1}=\Psi_{2}=0$. Then we have

$$
\begin{aligned}
\left\|u^{m}\right\|^{2} \leq & \left\|u^{0}\right\|^{2}+\frac{\tau^{\alpha}}{\Gamma(1+\alpha)}\left[(2-\alpha)^{2}+1\right]\left\|\delta_{x} u^{0}\right\|_{\varphi}^{2} \\
& +\frac{L^{2}}{12 c_{1}} \Gamma(1+\alpha) \tau^{2-\alpha} \cdot\left\|f^{1}\right\|^{2} \\
& +\frac{L^{2}}{6 c_{1}} \cdot \Gamma(\alpha) \tau \cdot T^{1-\alpha} \sum_{n=2}^{m}\left\|f^{n-\frac{1}{2}}\right\|^{2}
\end{aligned}
$$


Proof Taking the inner product of (49) with $u^{n-\frac{1}{2}}$, we have

$$
\begin{aligned}
\left(\delta_{t} u^{n-\frac{1}{2}}, u^{n-\frac{1}{2}}\right)= & \frac{\tau^{\alpha-1}}{\Gamma(1+\alpha)}\left[\left(\delta_{x}\left(\varphi \delta_{x} u\right)^{n-\frac{1}{2}}, u^{n-\frac{1}{2}}\right)-\sum_{k=1}^{n-1}\left(a_{n-k-1}-a_{n-k}\right)\right. \\
& \left.\cdot\left(\delta_{x}\left(\varphi \delta_{x} u\right)^{k-\frac{1}{2}}, u^{n-\frac{1}{2}}\right)+\hat{a}_{n-1}\left(\delta_{x}\left(\varphi \delta_{x} u\right)^{0}, u^{n-\frac{1}{2}}\right)\right] \\
& +\left(f^{n-\frac{1}{2}}, u^{n-\frac{1}{2}}\right) .
\end{aligned}
$$

Noticing that

$$
\left(\delta_{t} u^{n-\frac{1}{2}}, u^{n-\frac{1}{2}}\right)=\frac{1}{2 \tau}\left(\left\|u^{n}\right\|^{2}-\left\|u^{n-1}\right\|^{2}\right)
$$

and using the discrete Green formula and zero boundary conditions for every term in the right-hand side, we obtain

$$
\begin{aligned}
\frac{\left\|u^{n}\right\|^{2}-\left\|u^{n-1}\right\|^{2}}{2 \tau}= & \frac{\tau^{\alpha-1}}{\Gamma(1+\alpha)}\left[-\left(\delta_{x} u^{n-\frac{1}{2}}, \delta_{x} u^{n-\frac{1}{2}}\right)_{\varphi}+\sum_{k=1}^{n-1}\left(a_{n-k-1}-a_{n-k}\right)\right. \\
& \left.\cdot\left(\delta_{x} u^{k-\frac{1}{2}}, \delta_{x} u^{n-\frac{1}{2}}\right)_{\varphi}-\hat{a}_{n-1}\left(\delta_{x} u^{0}, \delta_{x} u^{n-\frac{1}{2}}\right)_{\varphi}\right] \\
& +\left(f^{n-\frac{1}{2}}, u^{n-\frac{1}{2}}\right) .
\end{aligned}
$$

Applying the Cauchy-Schwarz inequality, we have

$$
\begin{aligned}
\frac{\left\|u^{n}\right\|^{2}-\left\|u^{n-1}\right\|^{2}}{2 \tau} \leq & \frac{\tau^{\alpha-1}}{\Gamma(1+\alpha)}\left[-\left\|\delta_{x} u^{n-\frac{1}{2}}\right\|_{\varphi}^{2}+\frac{1}{2} \sum_{k=1}^{n-1}\left(a_{n-k-1}-a_{n-k}\right)\right. \\
& \left.\cdot\left(\left\|\delta_{x} u^{k-\frac{1}{2}}\right\|_{\varphi}^{2}+\left\|\delta_{x} u^{n-\frac{1}{2}}\right\|_{\varphi}^{2}\right)+\frac{\hat{a}_{n-1}}{2}\left(\left\|\delta_{x} u^{n-\frac{1}{2}}\right\|_{\varphi}^{2}+\left\|\delta_{x} u^{0}\right\|_{\varphi}^{2}\right)\right] \\
& +\left|\left(f^{n-\frac{1}{2}}, u^{n-\frac{1}{2}}\right)\right| .
\end{aligned}
$$

Letting $\frac{\tau^{r-1}}{\Gamma(1+r)}=I$, we have

$$
\begin{aligned}
\frac{\left\|u^{n}\right\|^{2}-\left\|u^{n-1}\right\|^{2}}{I \tau} \leq & -2\left\|\delta_{x} u^{n-\frac{1}{2}}\right\|_{\varphi}^{2}+\left(a_{0}-a_{n-1}\right)\left\|\delta_{x} u^{n-\frac{1}{2}}\right\|_{\varphi}^{2}+\frac{2}{I}\left|\left(f^{n-\frac{1}{2}}, u^{n-\frac{1}{2}}\right)\right| \\
& +\sum_{k=1}^{n-1}\left(a_{n-k-1}-a_{n-k}\right)\left\|\delta_{x} u^{k-\frac{1}{2}}\right\|_{\varphi}^{2}+\hat{a}_{n-1}\left(\left\|\delta_{x} u^{n-\frac{1}{2}}\right\|_{\varphi}^{2}+\left\|\delta_{x} u^{0}\right\|_{\varphi}^{2}\right) \\
= & \left(\hat{a}_{n-1}-a_{n-1}-1\right)\left\|\delta_{x} u^{n-\frac{1}{2}}\right\|_{\varphi}^{2} \\
& +\hat{a}_{n-1}\left\|\delta_{x} u^{0}\right\|_{\varphi}^{2}+\sum_{k=1}^{n-1}\left(a_{n-k-1}-a_{n-k}\right)\left\|\delta_{x} u^{k-\frac{1}{2}}\right\|_{\varphi}^{2} \\
& +\frac{2}{I}\left|\left(f^{n-\frac{1}{2}}, u^{n-\frac{1}{2}}\right)\right| .
\end{aligned}
$$


From (57) we know that

$$
\begin{aligned}
\frac{\left\|u^{n}\right\|^{2}-\left\|u^{n-1}\right\|^{2}}{I \tau} \leq & -\left\|\delta_{x} u^{n-\frac{1}{2}}\right\|_{\varphi}^{2}+\left(a_{n-2}-a_{n-1}\right)\left\|\delta_{x} u^{0}\right\|_{\varphi}^{2} \\
& +\sum_{k=1}^{n-1}\left(a_{n-k-1}-a_{n-k}\right)\left\|\delta_{x} u^{k-\frac{1}{2}}\right\|_{\varphi}^{2}+\frac{2}{I}\left|\left(f^{n-\frac{1}{2}}, u^{n-\frac{1}{2}}\right)\right| .
\end{aligned}
$$

Summing up for $2 \leq n \leq m$ and changing the summation order in the third term of the right-hand side, we get

$$
\begin{aligned}
\frac{\left\|u^{m}\right\|^{2}-\left\|u^{1}\right\|^{2} \leq}{I \tau}- & \sum_{n=2}^{m}\left\|\delta_{x} u^{n-\frac{1}{2}}\right\|_{\varphi}^{2}+\left(a_{0}-a_{m-1}\right)\left\|\delta_{x} u^{0}\right\|_{\varphi}^{2} \\
& +\sum_{k=1}^{m-1} \sum_{n=k+1}^{m}\left(a_{n-k-1}-a_{n-k}\right)\left\|\delta_{x} u^{k-\frac{1}{2}}\right\|_{\varphi}^{2}+\frac{2}{I} \sum_{n=2}^{m}\left|\left(f^{n-\frac{1}{2}}, u^{n-\frac{1}{2}}\right)\right| \\
= & -\sum_{k=2}^{m}\left\|\delta_{x} u^{k-\frac{1}{2}}\right\|_{\varphi}^{2}+\sum_{k=1}^{m-1}\left(a_{0}-a_{m-k}\right)\left\|\delta_{x} u^{k-\frac{1}{2}}\right\|_{\varphi}^{2} \\
& +\left(a_{0}-a_{m-1}\right)\left\|\delta_{x} u^{0}\right\|_{\varphi}^{2}+\frac{2}{I} \sum_{n=2}^{m}\left|\left(f^{n-\frac{1}{2}}, u^{n-\frac{1}{2}}\right)\right| \\
= & \sum_{k=2}^{m-1}\left(-a_{m-k}\right)\left\|\delta_{x} u^{k-\frac{1}{2}}\right\|_{\varphi}^{2}-\left\|\delta_{x} u^{m-\frac{1}{2}}\right\|_{\varphi}^{2}+\frac{2}{I} \sum_{n=2}^{m}\left|\left(f^{n-\frac{1}{2}}, u^{n-\frac{1}{2}}\right)\right| \\
& +\left(a_{0}-a_{m-1}\right)\left(\left\|\delta_{x} u^{\frac{1}{2}}\right\|_{\varphi}^{2}+\left\|\delta_{x} u^{0}\right\|_{\varphi}^{2}\right) \\
= & \sum_{k=2}^{m}\left(-a_{m-k}\right)\left\|\delta_{x} u^{k-\frac{1}{2}}\right\|_{\varphi}^{2}+\left(a_{0}-a_{m-1}\right)\left(\left\|\delta_{x} u^{\frac{1}{2}}\right\|_{\varphi}^{2}+\left\|\delta_{x} u^{0}\right\|_{\varphi}^{2}\right) \\
& +\frac{2}{I} \sum_{n=2}^{m}\left|\left(f^{n-\frac{1}{2}}, u^{n-\frac{1}{2}}\right)\right| .
\end{aligned}
$$

Using the Cauchy inequality, (53), and Lemma 1, we have

$$
\frac{2}{I}\left|\left(f^{n-\frac{1}{2}}, u^{n-\frac{1}{2}}\right)\right| \leq \frac{L^{2}}{6 c_{1} I^{2} a_{m-n}}\left\|f^{n-\frac{1}{2}}\right\|^{2}+a_{m-n}\left\|\delta_{x} u^{n-\frac{1}{2}}\right\|_{\varphi}^{2}
$$

Substituting (67) into (66), we have

$$
\left\|u^{m}\right\|^{2} \leq\left\|u^{1}\right\|^{2}+I \tau\left(a_{0}-a_{m-1}\right)\left(\left\|\delta_{x} u^{\frac{1}{2}}\right\|_{\varphi}^{2}+\left\|\delta_{x} u^{0}\right\|_{\varphi}^{2}\right)+\sum_{n=2}^{m} \frac{\tau L^{2}}{6 c_{1} I a_{m-n}}\left\|f^{n-\frac{1}{2}}\right\|^{2}
$$

Taking the inner product of (50) with $u^{n-\frac{1}{2}}$, we have

$$
\begin{aligned}
\frac{\left\|u^{1}\right\|^{2}-\left\|u^{0}\right\|^{2}}{2 \tau} & =I\left[-\left(\delta_{x} u^{1}, \delta_{x} u^{\frac{1}{2}}\right)_{\varphi}+(1-\alpha)\left(\delta_{x} u^{0}, \delta_{x} u^{\frac{1}{2}}\right)_{\varphi}\right]+\left(f^{1}, u^{\frac{1}{2}}\right) \\
& =I\left[-2\left\|\delta_{x} u^{\frac{1}{2}}\right\|_{\varphi}^{2}+(2-\alpha)\left(\delta_{x} u^{0}, \delta_{x} u^{\frac{1}{2}}\right)_{\varphi}\right]+\left(f^{1}, u^{\frac{1}{2}}\right),
\end{aligned}
$$


so that

$$
\left\|u^{1}\right\|^{2}=\left\|u^{0}\right\|^{2}+I \tau\left[-4\left\|\delta_{x} u^{\frac{1}{2}}\right\|_{\varphi}^{2}+2(2-\alpha)\left(\delta_{x} u^{0}, \delta_{x} u^{\frac{1}{2}}\right)_{\varphi}\right]+2 \tau\left(f^{1}, u^{\frac{1}{2}}\right) .
$$

Substituting (69) into (68) and using the Cauchy-Schwarz inequality again, we arrive at

$$
\begin{aligned}
\left\|u^{m}\right\|^{2} \leq & \left\|u^{0}\right\|^{2}+I \tau\left[-3\left\|\delta_{x} u^{\frac{1}{2}}\right\|_{\varphi}^{2}+2(2-\alpha)\left(\delta_{x} u^{0}, \delta_{x} u^{\frac{1}{2}}\right)_{\varphi}\right] \\
& +I \tau a_{0}\left\|\delta_{x} u^{0}\right\|_{\varphi}^{2}+\sum_{n=2}^{m} \frac{\tau L^{2}}{6 c_{1} I a_{m-n}}\left\|f^{n-\frac{1}{2}}\right\|^{2}+2 \tau\left(f^{1}, u^{\frac{1}{2}}\right) \\
\leq & \left\|u^{0}\right\|^{2}-2 I \tau\left\|\delta_{x} u^{\frac{1}{2}}\right\|_{\varphi}^{2}+I \tau\left[(2-\alpha)^{2}+1\right]\left\|\delta_{x} u^{0}\right\|_{\varphi}^{2} \\
& +\sum_{n=2}^{m} \frac{\tau L^{2}}{6 c_{1} I a_{m-n}}\left\|f^{n-\frac{1}{2}}\right\|^{2}+2 I \tau\left\|\delta_{x} u^{\frac{1}{2}}\right\|_{\varphi}^{2}+\frac{L^{2} \tau}{12 I c_{1}}\left\|f^{1}\right\|^{2} .
\end{aligned}
$$

Noticing that $a_{m-n}>a_{m-1}>\alpha m^{\alpha-1}>\alpha N^{\alpha-1}$, we have

$$
\begin{aligned}
\sum_{n=2}^{m} \frac{\tau L^{2}}{6 c_{1} I a_{m-n}}\left\|f^{n-\frac{1}{2}}\right\|^{2} & \leq \frac{\tau L^{2}}{6 c_{1} I \alpha N^{\alpha-1}} \sum_{n=2}^{m}\left\|f^{n-\frac{1}{2}}\right\|^{2} \\
& =\frac{L^{2}}{6 c_{1}} \cdot \frac{\tau^{2-\alpha} \Gamma(1+\alpha)}{\alpha N^{\alpha-1}} \sum_{n=2}^{m}\left\|f^{n-\frac{1}{2}}\right\|^{2} \\
& =\frac{L^{2}}{6 c_{1}} \cdot \Gamma(\alpha) \tau \cdot T^{1-\alpha} \sum_{n=2}^{m}\left\|f^{n-\frac{1}{2}}\right\|^{2} .
\end{aligned}
$$

Then, substituting (71) into (70), we get (61) for $2 \leq m \leq N$, and (61) for $m=1$ is obvious. So the proof is completed.

\subsection{Convergence}

We now consider the convergence of the difference scheme (49)-(52). Let

$$
e_{i}^{n}=U_{i}^{n}-u_{i}^{n}, \quad 0 \leq i \leq M, 0 \leq n \leq N .
$$

Subtracting (49)-(52) from (41)-(44) and (47)-(48), we get the equations for the error:

$$
\begin{aligned}
& \delta_{t} e_{i}^{n-\frac{1}{2}}=\frac{\tau^{\alpha-1}}{\Gamma(1+\alpha)}\left[\delta_{x}\left(\varphi \delta_{x} e\right)_{i}^{n-\frac{1}{2}}-\sum_{k=1}^{n-1}\left(a_{n-k-1}-a_{n-k}\right) \delta_{x}\left(\varphi \delta_{x} e\right)_{i}^{k-\frac{1}{2}}\right]+R_{i}^{n}, \\
& 1 \leq i \leq M-1,2 \leq n \leq N, \\
& \delta_{t} e_{i}^{\frac{1}{2}}=\frac{\tau^{\alpha-1}}{\Gamma(1+\alpha)} \delta_{x}\left(\varphi \delta_{x} e\right)_{i}^{1}+R_{i}, \quad 1 \leq i \leq M-1, \\
& e_{0}^{n}=0, \quad e_{M}^{n}=0, \quad 1 \leq n \leq N, \\
& e_{i}^{0}=0, \quad 0 \leq i \leq M .
\end{aligned}
$$


Then from Theorem 1 we have

$$
\left\|e^{n}\right\|^{2} \leq \frac{L^{2}}{12 c_{1}} \Gamma(1+\alpha) \tau^{2-\alpha} \cdot\|R\|^{2}+\frac{L^{2}}{6 c_{1}} \tau \Gamma(\alpha) T^{1-\alpha} \cdot \sum_{k=2}^{n}\left\|R^{k}\right\|^{2} .
$$

From (45) and (46) we have

$$
\begin{aligned}
\left\|e^{n}\right\|^{2} & \leq \frac{L^{3}}{12 c_{1}} \Gamma(1+\alpha) \tau^{2-\alpha}\left[c\left(\tau+h^{2}\right)\right]^{2}+\frac{L^{2}}{6 c_{1}} N \tau \Gamma(\alpha) T^{1-\alpha}\left[c\left(\tau^{1+\alpha}+h^{2}\right)\right]^{2} \\
& \leq \frac{L^{3}}{12 c_{1}} \Gamma(1+\alpha)\left[c\left(\tau^{2-\frac{\alpha}{2}}+h^{2}\right)\right]^{2}+\frac{L^{2}}{6 c_{1}} \Gamma(\alpha) T^{2-\alpha}\left[c\left(\tau^{1+\alpha}+h^{2}\right)\right]^{2} .
\end{aligned}
$$

Letting

$$
\beta=\min \left\{2-\frac{\alpha}{2}, 1+\alpha\right\}, \quad \hat{c}=L c \sqrt{\frac{\Gamma(\alpha)}{12 c_{1}}\left(L \alpha+2 T^{2-\alpha}\right)},
$$

we have the convergence in the $L_{2}$ norm.

Theorem 2 Suppose that problem (10)-(12) has a smooth solution $u(x, t)$ in the domain $[0, L] \times[0, T]$ and $u_{i}^{n}, 0 \leq i \leq M, 1 \leq n \leq N$, is a solution of the difference scheme (49)-(52).

Then

$$
\max _{1 \leq n \leq N}\left\|u\left(x_{i}, t^{n}\right)-u_{i}^{n}\right\| \leq \hat{c}\left(\tau^{\beta}+h^{2}\right)
$$

Remark The Crank-Nicolson-type scheme involves two time levels for a RiemannLiouville fractional subdiffusion equation with spatially variable coefficient. Therefore, we actually used recursion method to handle the analysis of stability. Then we get the convergence, and the convergence order in spatial direction is just two. It is difficult to improve the space accuracy by introducing a compact technique.

\section{Numerical examples}

In this section, we give two examples to testify the efficiency and convergence orders of our difference scheme.

Example 1 Consider the following problem with zero initial value:

$$
\begin{aligned}
& \frac{\partial u(x, t)}{\partial t}={ }_{0} \mathcal{D}_{t}^{1-\alpha}\left\{\frac{\partial}{\partial x}\left[\left(x^{2}+1\right) \frac{\partial u}{\partial x}\right]\right\}+e^{x}(2+\alpha) t^{1+\alpha} \\
& \quad-e^{x}(x+1)^{2} \cdot \frac{\Gamma(3+\alpha)}{\Gamma(2+2 \alpha)}, \quad 0<x<1,0<t \leq 1, \\
& u(0, t)=t^{2+\alpha}, \quad u(1, t)=e t^{2+\alpha}, \quad 0<t \leq 1, \\
& u(x, 0)=0, \quad 0 \leq x \leq 1 .
\end{aligned}
$$

The exact solution is $u(x, t)=e^{x} t^{2+\alpha}$. 
Table 1 The maximum $L_{2}$ errors and convergence orders for Example 1 where $h=1 / 1,000$

\begin{tabular}{llll}
\hline $\boldsymbol{\alpha}$ & $\boldsymbol{\tau}$ & $\boldsymbol{E}_{\boldsymbol{L}_{\mathbf{2}}}$ & $\operatorname{Order}(\boldsymbol{\tau} \boldsymbol{)}$ \\
\hline $1 / 3$ & $1 / 20$ & $3.6326 \mathrm{e}-003$ & $*$ \\
& $1 / 40$ & $1.4826 \mathrm{e}-003$ & $1.2929 \mathrm{e}+000$ \\
& $1 / 80$ & $5.9838 \mathrm{e}-004$ & $1.3090 \mathrm{e}+000$ \\
& $1 / 160$ & $2.3989 \mathrm{e}-004$ & $1.3187 \mathrm{e}+000$ \\
0.5 & $1 / 20$ & $1.8364 \mathrm{e}-003$ & $*$ \\
& $1 / 40$ & $6.8187 \mathrm{e}-004$ & $1.4293 \mathrm{e}+000$ \\
& $1 / 80$ & $2.4895 \mathrm{e}-004$ & $1.4536 \mathrm{e}+000$ \\
& $1 / 160$ & $8.9904 \mathrm{e}-005$ & $1.4694 \mathrm{e}+000$ \\
& & & \\
& $1 / 3$ & $8.0971 \mathrm{e}-004$ & $*$ \\
& $1 / 20$ & $2.7806 \mathrm{e}-004$ & $1.5420 \mathrm{e}+000$ \\
& $1 / 80$ & $9.3144 \mathrm{e}-005$ & $1.5779 \mathrm{e}+000$ \\
& $1 / 160$ & $3.0673 \mathrm{e}-005$ & $1.6025 \mathrm{e}+000$ \\
\hline
\end{tabular}

Table 2 The maximum $L_{2}$ errors and convergence orders for Example 1 where $\tau=1 / 10,000$

\begin{tabular}{llll}
\hline $\boldsymbol{\alpha}$ & $\boldsymbol{h}$ & $\boldsymbol{E}_{\boldsymbol{L}_{\mathbf{2}}}$ & $\operatorname{Order}(\boldsymbol{h})$ \\
\hline $1 / 3$ & $1 / 4$ & $3.6326 \mathrm{e}-003$ & $*$ \\
& $1 / 8$ & $8.4541 \mathrm{e}-004$ & $2.1033 \mathrm{e}+000$ \\
& $1 / 16$ & $2.1080 \mathrm{e}-004$ & $2.0038 \mathrm{e}+000$ \\
& $1 / 32$ & $5.1974 \mathrm{e}-005$ & $2.0200 \mathrm{e}+000$ \\
0.5 & $1 / 4$ & $3.3024 \mathrm{e}-003$ & $*$ \\
& $1 / 8$ & $8.3002 \mathrm{e}-004$ & $1.9923 \mathrm{e}+000$ \\
& $1 / 16$ & $2.0758 \mathrm{e}-004$ & $1.9995 \mathrm{e}+000$ \\
& $1 / 32$ & $5.1766 \mathrm{e}-005$ & $2.0036 \mathrm{e}+000$ \\
& $1 / 3$ & & \\
& $1 / 4$ & $3.2191 \mathrm{e}-003$ & $*$ \\
& $1 / 8$ & $8.1000 \mathrm{e}-004$ & $1.9907 \mathrm{e}+000$ \\
& $1 / 16$ & $2.0274 \mathrm{e}-004$ & $1.9983 \mathrm{e}+000$ \\
& $1 / 32$ & $5.0674 \mathrm{e}-005$ & $2.0003 \mathrm{e}+000$ \\
\hline
\end{tabular}

Let

$$
\begin{aligned}
& E_{L_{2}}(h, \tau)=\max _{1 \leq n \leq N}\left\|u^{n}-U^{n}\right\|, \\
& \operatorname{Order}(\tau)=\log _{2}\left(\frac{E_{L_{2}}(h, 2 \tau)}{E_{L_{2}}(h, \tau)}\right), \quad \operatorname{Order}(h)=\log _{2}\left(\frac{E_{L_{2}}(2 h, \tau)}{E_{L_{2}}(h, \tau)}\right) .
\end{aligned}
$$

We solve problem (80)-(82) with the Crank-Nicolson-type scheme (49)-(52). Fixing the spatial step $h=1 / 1,000$ and taking different temporal steps, Table 1 presents the maximum $L_{2}$ norm errors and convergence orders of our schemes; fixing the temporal step $\tau=1 / 10,000$ and taking different spatial steps, Table 2 presents the $L_{2}$ norm errors and convergence orders in spatial direction. In both cases, we take $\alpha$ to be $1 / 3,1 / 2,2 / 3$. The results show that the Crank-Nicolson-type scheme has accuracy of order $1+\alpha$ in the temporal direction and order 2 in the spatial direction.

Example 2 Now we give a problem with nonzero initial value:

$$
\begin{aligned}
\frac{\partial u(x, t)}{\partial t}= & { }_{0} \mathcal{D}_{t}^{1-\alpha}\left\{\frac{\partial}{\partial x}\left[\left(x^{2}+1\right) \frac{\partial u}{\partial x}\right]\right\}+\cos (\pi x) \cdot(3+\alpha) t^{2+\alpha} \\
& +\left[2 \pi x \sin (\pi x)+\pi^{2}\left(x^{2}+1\right) \cos (\pi x)\right]
\end{aligned}
$$


Table 3 The maximum $L_{2}$ errors and convergence orders for Example 2 with $h=1 / 1,000$

\begin{tabular}{|c|c|c|c|}
\hline$\alpha$ & $\tau$ & $E_{L_{2}}$ & $\operatorname{Order}(\tau)$ \\
\hline \multirow[t]{4}{*}{$1 / 3$} & $1 / 20$ & $4.5529 \mathrm{e}-003$ & $*$ \\
\hline & $1 / 40$ & $1.8482 \mathrm{e}-003$ & $1.3007 \mathrm{e}+000$ \\
\hline & $1 / 80$ & $7.4443 e-004$ & $1.3119 \mathrm{e}+000$ \\
\hline & $1 / 160$ & $2.9840 \mathrm{e}-004$ & $1.3189 \mathrm{e}+000$ \\
\hline \multirow[t]{4}{*}{0.5} & $1 / 20$ & $2.4404 \mathrm{e}-003$ & * \\
\hline & $1 / 40$ & $8.9749 e-004$ & $1.4431 \mathrm{e}+000$ \\
\hline & $1 / 80$ & $3.2613 e-004$ & $1.4604 \mathrm{e}+000$ \\
\hline & $1 / 160$ & $1.1768 \mathrm{e}-004$ & $1.4706 \mathrm{e}+000$ \\
\hline \multirow[t]{4}{*}{$2 / 3$} & $1 / 20$ & $1.1972 \mathrm{e}-003$ & $*$ \\
\hline & $1 / 40$ & $4.0070 \mathrm{e}-004$ & $1.5791 \mathrm{e}+000$ \\
\hline & $1 / 80$ & $1.3221 \mathrm{e}-004$ & $1.5997 \mathrm{e}+000$ \\
\hline & $1 / 160$ & $4.3321 \mathrm{e}-005$ & $1.6097 \mathrm{e}+000$ \\
\hline
\end{tabular}

Table 4 The maximum $L_{2}$ errors and convergence orders for Example 2 with $\tau=1 / 10,000$

\begin{tabular}{llll}
\hline $\boldsymbol{\alpha}$ & $\boldsymbol{h}$ & $\boldsymbol{E}_{\boldsymbol{L}_{\mathbf{2}}}$ & $\operatorname{Order}(\boldsymbol{h})$ \\
\hline $1 / 3$ & $1 / 4$ & $2.9470 \mathrm{e}-002$ & $*$ \\
& $1 / 8$ & $7.1231 \mathrm{e}-003$ & $2.0487 \mathrm{e}+000$ \\
& $1 / 16$ & $1.7659 \mathrm{e}-003$ & $2.0121 \mathrm{e}+000$ \\
& $1 / 32$ & $4.4129 \mathrm{e}-004$ & $2.0006 \mathrm{e}+000$ \\
0.5 & $1 / 4$ & $2.9634 \mathrm{e}-002$ & $*$ \\
& $1 / 8$ & $7.1649 \mathrm{e}-003$ & $2.0482 \mathrm{e}+000$ \\
& $1 / 16$ & $1.7757 \mathrm{e}-003$ & $2.0126 \mathrm{e}+000$ \\
& $1 / 32$ & $4.4311 \mathrm{e}-004$ & $2.0027 \mathrm{e}+000$ \\
& & & \\
& $1 / 3$ & $2.9400 \mathrm{e}-002$ & $*$ \\
& $1 / 4$ & $7.1113 \mathrm{e}-003$ & $2.0476 \mathrm{e}+000$ \\
& $1 / 16$ & $1.7625 \mathrm{e}-003$ & $2.0125 \mathrm{e}+000$ \\
& $1 / 32$ & $4.3969 \mathrm{e}-004$ & $2.0031 \mathrm{e}+000$ \\
\end{tabular}

$$
\begin{gathered}
\cdot\left[\frac{\Gamma(4+\alpha)}{\Gamma(3+2 \alpha)} t^{2+2 \alpha}+\frac{1}{\Gamma(\alpha)} t^{\alpha-1}\right], \quad 0<x<1,0<t \leq 1, \\
u(0, t)=t^{3+\alpha}+1, \quad u(1, t)=-t^{3+\alpha}-1, \quad 0<t \leq 1, \\
u(x, 0)=\cos (\pi x), \quad 0 \leq x \leq 1 .
\end{gathered}
$$

The exact solution is $u(x, t)=\cos (\pi x)\left(t^{3+\alpha}+1\right)$. We solve problem (83)-(85) with the Crank-Nicolson-type scheme (49)-(52) and present the numerical results in Tables 3 and 4 . The results show that our scheme is still efficient for nonzero initial value problems. In Figures 1 and 2, we plot surface figures of the error $\left(\left|u\left(x_{i}, t_{n}\right)-u_{i}^{n}\right|\right)$ with different mesh sizes when $\alpha=0.1,0.9$. These figures show that the maximum error becomes relatively smaller as the mesh size becomes smaller, which provides the validation of our results once more.

\section{Conclusion}

In this paper, we have presented a Crank-Nicolson-type difference scheme for the Riemann-Liouville fractional spatial variable coefficient subdiffusion equation. Based on the Crank-Nicolson technique and $L_{1}$ formula on the temporal direction, we proved that our difference scheme is unconditionally stable with respect to the initial value and the inhomogeneous term, and the numerical solution is convergent in the discrete $L_{2}$ norm. The convergence order is $\min \left\{2-\frac{\alpha}{2}, 1+\alpha\right\}$ in the temporal direction and two in the spa- 

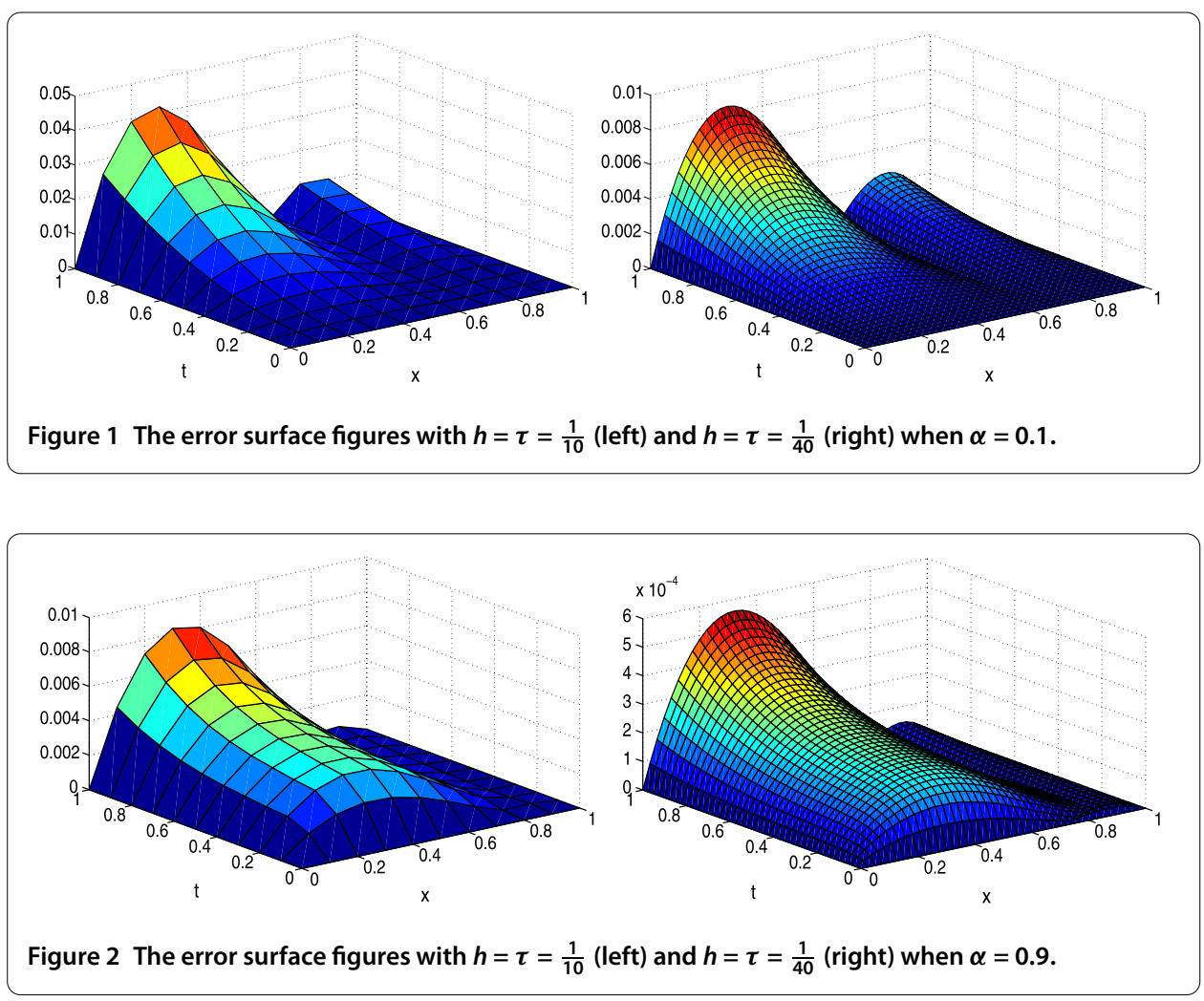

tial direction. This scheme results in a linear system in which the coefficient matrix is a tridiagonal and strictly diagonally dominant, so it can be solved by the Thomas algorithm. Two numerical examples are given to show the efficiency of the method. It is meaningful to construct a second-order difference scheme of this type, which will be our work in the future.

\section{Competing interests}

The authors declare that there is no conflict of interests regarding the publication of this paper.

Authors' contributions

Both authors contributed equally to the writing of this paper. Both authors read and approved the final manuscript.

\section{Author details}

${ }^{1}$ State Key Laboratory for Geomechanics and Deep Underground Engineering, China University of Mining and Technology, Xuzhou, Jiangsu 221116, P.R. China. ${ }^{2}$ School of Basic Education Sciences, Xuzhou Medical University, Xuzhou, Jiangsu 221004, P.R. China. ${ }^{3}$ School of Mechanics and Civil Engineering, China University of Mining and Technology, Xuzhou, Jiangsu 221116, P.R. China.

\section{Acknowledgements}

We wish to thank the reviewers for their constructive comments that led to the improvement of the original manuscript. Financial support for this work was provided by the National Basic Research Program of China (Nos. 2015CB251601, 2013CB227900), National Natural Science Foundation (Nos. 51322401, 51421003, U1261201), the Fundamental Research Funds for the Central Universities (Nos. 2014YC09, 2014ZDPY08) (China University of Mining and Technology), and the 111 Project (No. B07028).

Received: 23 October 2016 Accepted: 17 January 2017 Published online: 23 January 2017

\section{References}

1. Podlubny, I: Fractional Differential Equations. Academic Press, San Diego (1999)

2. Metzler, R, Klafter, J: The random walk's guide to anomalous diffusion: a fractional dynamics approach. Phys. Rep. 339, 1-77 (2000)

3. Awotunde, AA, Ghanam, RA, Tatar, N-e: Artificial boundary condition for a modified fractional diffusion problem. Bound. Value Probl. 2015, 20 (2015) 
4. Zhang, L, Li, S: Regularity of weak solutions of the Cauchy problem to a fractional porous medium equation. Bound. Value Probl. 2015, 28 (2015)

5. Povstenko, Y, Klekot, J: The Dirichlet problem for the time-fractional advection-diffusion equation in a line segment. Bound. Value Probl. 2016, 89 (2016)

6. $\mathrm{Wu}, \mathrm{J}, \mathrm{Zhang}, \mathrm{X}, \mathrm{Liu}, \mathrm{L}, \mathrm{Wu}, \mathrm{Y}$ : Twin iterative solutions for a fractional differential turbulent flow model. Bound. Value Probl. 2016, 98 (2016)

7. Balakrishnan, V: Anomalous diffusion in one dimension. Physica A 132, 569-580 (1985)

8. Schneider, WR, Wyss, W: Fractional diffusion and wave equations. J. Math. Phys. 30, 134-144 (1989)

9. Marin, M: Some basic theorems in elastostatics of micropolar materials with voids. J. Comput. Appl. Math. 70, 115-126 (1996)

10. Marin, M, Marinescu, C: Thermoelasticity of initially stressed bodies, asymptotic equipartition of energies. Int. J. Eng. Sci. 36(1), 73-86 (1998)

11. Hameed, M, Khan, AA, Ellahi, R, Raza, M: Study of magnetic and heat transfer on the peristaltic transport of a fractional second grade fluid in a vertical tube. Eng. Sci. Technol. Int. J. 18(3), 496-502 (2015)

12. Langlands, TAM, Henry, BI: The accuracy and stability of an implicit solution method for the fractional diffusion equation. J. Comput. Phys. 205, 719-736 (2005)

13. Zhuang, P, Liu, F, Anh, V, Turner, I: New solution and analytical techniques of the implicit numerical method for the anomalous subdiffusion equation. SIAM J. Numer. Anal. 46, 1079-1095 (2008)

14. Yuste, SB, Acedo, L: An explicit finite difference method and a new von Neumann-type stability analysis for fractional diffusion equations. SIAM J. Numer. Anal. 42, 1862-1874 (2005)

15. Yuste, S: Weighted average finite difference methods for fractional diffusion equations. J. Comput. Phys. 216, 264-274 (2006)

16. Sun, ZZ, Wu, XN: A fully discrete difference scheme for a diffusion-wave system. Appl. Numer. Math. 56, 193-209 (2006)

17. Lin, $X, X u, C$ : Finite difference/spectral approximations for the time-fractional diffusion equation. J. Comput. Phys. 225, 1533-1552 (2007)

18. Chen, CM, Liu, F, Turner, I, Anh, V: A Fourier method for the fractional diffusion equation describing subdiffusion. J. Comput. Phys. 227, 886-897 (2007)

19. Gao, GH, Sun, ZZ: A compact difference scheme for the fractional subdiffusion equations. J. Comput. Phys. 230, 586-595 (2011)

20. Tian, WY, Zhou, H, Deng, WH: A class of second order difference approximations for solving space fractional diffusion equations. Math. Comput. 84, 1703-1727 (2015)

21. Li, C, Deng, WH: Second order WSGD operators II: a new family of difference schemes for space fractional advection diffusion equation (2013). arXiv:1310.7671v1 [math.NA]

22. Zhang, YN, Sun, ZZ, Wu, HW: Error estimates of Crank-Nicolson type difference schemes for the subdiffusion equation. SIAM J. Numer. Anal. 49, 2302-2322 (2011)

23. Wang, Z, Vong, S: Compact difference schemes for the modified anomalous fractional subdiffusion equation and the fractional diffusion-wave equation. J. Comput. Phys. 277, 1-15 (2014)

24. Wang, D, Xiao, A, Yang, W: Crank-Nicolson difference scheme for the coupled nonlinear Schrödinger equations with the Riesz space fractional derivative. J. Comput. Phys. 242, 670-681 (2013)

25. Zhao, X, Sun, ZZ: Compact Crank-Nicolson schemes for a class of fractional Cattaneo equation in inhomogeneous medium. J. Sci. Comput. 62(3), 747-771 (2014)

26. Sweilam, NH, Moharram, H, Moniem, NKA, Ahmed, S: A parallel Crank-Nicolson finite difference method for time-fractional parabolic equation. J. Numer. Math. 22(4), 363-382 (2014)

27. Ozbilge, E, Demir, A: Semigroup approach for identification of the unknown diffusion coefficient in a linear parabolic equation with mixed output data. Bound. Value Probl. 2013, 43 (2013)

28. Ozbilge, E, Demir, A: Analysis of the inverse problem in a time fractional parabolic equation with mixed boundary conditions. Bound. Value Probl. 2014, 134 (2014)

29. Demir, A, Kanca, F, Ozbilge, E: Numerical solution and distinguishability in time fractional parabolic equation. Bound. Value Probl. 2015, $142(2015)$

30. Zhao, X, Xu, Q: Efficient numerical schemes for fractional sub-diffusion equation with the spatially variable coefficient. Appl. Math. Model. 38(15-16), 3848-3859 (2014)

31. Vong, S, Lyu, P, Wang, Z: A compact difference scheme for fractional sub-diffusion equations with the spatially variable coefficient under Neumann boundary conditions. J. Sci. Comput. 66(2), 725-739 (2015)

32. Metzler, R, Glöckle, WG, Nonnenmacher, TF: Fractional model equation for anomalous diffusion. Physica A 211, 13-24 (1994)

33. Zeng, F, Li, C, Liu, F, Turner, I: The use of finite difference/element approaches for solving the time-fractional subdiffusion equation. SIAM J. Sci. Comput. 35(6), A2976-A3000 (2013)

34. Sun, ZZ: The Method of Order Reduction and Its Application to the Numerical Solutions of Partial Differential Equations. Science Press, Beijing (2009)

35. Sun, ZZ: Numerical Methods of Partial Differential Equations, 2nd edn. Science Press, Beijing (2012) (in Chinese) 